ARTICLE

\title{
Structure of the class C orphan GPCR GPR158 in complex with RGS7-G $\beta 5$
}

\author{
Eunyoung Jeong (i] ${ }^{1,2}$, Yoojoong Kim (1) ${ }^{1,2}$, Jihong Jeong (1) ${ }^{1} \&$ Yunje Cho (1) ${ }^{1 凶}$
}

GPR158, a class C orphan GPCR, functions in cognition, stress-induced mood control, and synaptic development. Among class C GPCRs, GPR158 is unique as it lacks a Venus flytrapfold ligand-binding domain and terminates Goi/o protein signaling through the RGS7-G $\beta 5$ heterodimer. Here, we report the cryo-EM structures of GPR158 alone and in complex with one or two RGS7-G $\beta 5$ heterodimers. GPR158 dimerizes through Per-Arnt-Sim-fold extracellular and transmembrane (TM) domains connected by an epidermal growth factor-like linker. The TM domain (TMD) reflects both inactive and active states of other class C GPCRs: a compact intracellular TMD, conformations of the two intracellular loops (ICLs) and the TMD interface formed by TM4/5. The ICL2, ICL3, TM3, and first helix of the cytoplasmic coiled-coil provide a platform for the DHEX domain of one RGS7 and the second helix recruits another RGS7. The unique features of the RGS7-binding site underlie the selectivity of GPR158 for RGS7.

\footnotetext{
${ }^{1}$ Department of Life Science, Pohang University of Science and Technology, Pohang, Republic of Korea. ${ }^{2}$ These authors contributed equally: Eunyoung Jeong,

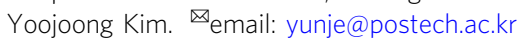


G PR158 is a class $C$ orphan $G$ protein-coupled receptor (GPCR) prominently expressed in brain tissue ${ }^{1}$. Although the pathological function of GPR158 is not clearly understood, the receptor is believed to play important roles in memory, stress-related mood control, and synaptogenesis ${ }^{2-4}$. In the CA3 region of the hippocampus of mice, GPR158 transduces the osteocalcin $(\mathrm{OCN})$ signal and enhances memory, in part through inositol 1,4,5-trisphosphate (IP3) and brain-derived neurotrophic factor ${ }^{2}$. Increased levels of GPR158 have been observed in major depressive disorder patients and chronic stressed mice, whereas ablation of GPR158 in mice produced antidepressant-like affects $^{3}$. GPR158 forms a trans-synaptic complex with proteoglycans of the extracellular matrix and controls the presynaptic differentiation of mossy fiber-CA3 synapses $^{4}$. In addition, GPR158 is implicated in the development of prostate cancer ${ }^{5}$.

The class C GPCR family comprises $\mathrm{Ca}^{2+}$-sensing (CaSR), metabotropic glutamate receptors (mGluR1-8), $\gamma$-aminobutyric acid receptor $\mathrm{B}\left(\mathrm{GABA}_{\mathrm{B}}\right)$, and sweet taste receptors, which are characterized by a large extracellular domain and dimerization ${ }^{6}$. Two orphan GPCRs, GPR158 and GPR179, are the least characterized class $\mathrm{C}$ members, and share over $70 \%$ sequence similarity in both extracellular and TM domains ${ }^{1}$. Unlike other class C GPCRs, GPR158 and GPR179 devoid of a Venus flytrap (VFT)-fold in their extracellular domain, and transmit signals via noncanonical mechanism by which both GPCRs recruit the regulator of $G$ protein signaling 7 (RGS7)-G $\beta 5$ heterodimer to the plasma membrane $e^{1,7,8}$. Activation of the $G$ protein by GPR158 remains unclear: although OCN binds to a complex containing GPR158 and Gaq and regulates the IP3 production, no functional assay using OCN as an agonist has been reported ${ }^{2}$. By contrast, GPR158 exhibits constitutive activity for Gi/o proteins but not for $\mathrm{Gq}^{9}$. The noncanonical signaling for GPR158 is more clearly established: GPR158 localizes RGS7-G 35 and the Gai/o protein activated by other GPCRs, and allosterically promotes GTPase activity of Gai/o, which ultimately reduces the activity of adenylate cyclase and controls other signaling pathways $7,8,10,11$. Because signal termination is a sensitive biological event, a GPCR must recognize RGS proteins with high specificity ${ }^{12-18}$. However, despite recent progress in GPCR structural biology, it remains unresolved how a GPCR specifically binds to RGS proteins at the molecular level ${ }^{19}$. Here, we report the structures of GPR158 alone $(3.5 \AA)$ and in complex with one or two RGS7-G $\beta 5$ heterodimers $(4.3,4.7 \AA)$, and provide insights into the noncanonical signaling mechanism by which the orphan GPCRs selectively recruit the RGS7-Gß5 complex and regulate the signals.

\section{Results}

Overall structure. We determined the overall structure of apo GPR158 at an average resolution of $3.5 \AA$ (Supplementary Figs. 1 and 2 and Supplementary Table 1). GPR158 consists of an $\alpha / \beta$ fold extracellular domain, a cysteine-rich (CR) domain with an epidermal growth factor (EGF)-like fold, and 7TM domain. GPR158 forms an elongated shape $\left(79 \times 147 \times 49 \AA^{3}\right)$ and dimerizes through both extracellular and TM domains (Fig. 1). Each domain of a GPR158 protomer is nearly identical to the corresponding part of another protomer with root-mean-square deviation (r.m.s.d.) ranging from 0.5 to $1.1 \AA$. However, entire GPR158 protomers differ significantly with an r.m.s.d. of $3.3 \AA$, indicating the flexibility between the domains within a protomer (Supplementary Fig. 1h). Overall, the GPR158 structure adopts a unique topology in extracellular and linker domains as well as its TM interface arrangement, different from other class C GPCRs $^{6}$.
Structure of the extracellular domain. The extracellular domain consists of the N-terminal three helices and the core. The core is comprised of a six-stranded sheet at the center, two vertically aligned helices on one face, and a horizontally aligned helix on another face (Fig. 2a, b). A DALI search revealed that the core structure most resembles the Per-Arnt-Sim (PAS) domain that is frequently observed in signaling proteins related to circadian, light and oxygen sensing ${ }^{20,21}$. Structural comparison with various PAS domains showed that the extracellular core of GPR158 has the r.m.s.d. values of 2.1-2.5 $\AA$ for $145 \mathrm{Ca}$ atoms with transducerlike protein 3 (Tlp3), histidine kinase (HK)-Z3, and HK-Z6 (refs. 22,23) (Supplementary Fig. 3).

The extracellular domain dimerizes through the side-by-side packing of $\alpha 4$ and $\alpha 5^{\prime}$ and $\alpha 5$ and $\alpha 4^{\prime}$ helices (' indicates the second protomer) in a parallel manner, with a buried surface area of $1806 \AA^{2}$ (Fig. 2a, c and Supplementary Fig. 4a). At the center of the interface, a hydrophobic cluster formed from $\mathrm{W} 156^{\prime}$ and L160' $\left(\alpha 5^{\prime}\right)$ and M139 and F135 ( $\left.\alpha 4\right)$ is packed against a symmetrical hydrophobic cluster, and the resulting hydrophobic network stabilizes the extracellular dimer. Below the PAS domain, the N-terminal three antiparallel helices are placed at the midpoint while forming close contacts with both PAS and CR domains (Figs. 1c and 2a). The three helices do not interact with the PAS core. Instead, helix $\alpha 2$ interacts with the $\beta 4-\beta 5$ loop and a B loop of the CR linker. The CR linker consists of two parts: the top two-thirds (A-C loops) folds into an EGF-like structure and the bottom third (D loop) forms a spiral-loop structure (Fig. 2d). The EGF-like and spiral motifs are stabilized through three and two disulfide bonds, respectively, which are also conserved in GPR179 (Fig. 2d and Supplementary Figs. 4b and 5a). The EGFlike linker can be superimposed to other EGF-like domains with r.m.s.d. values of 2.0-2.3 $\AA$ for $66 \mathrm{Ca}$ atoms ${ }^{24-27}$ (Supplementary Fig. 5b, c). A pairwise comparison with the Factor IX EGF-like domain reveals that the conserved D314 and D316 (A loop) and the main chain oxygen atoms of the elongated $\mathrm{B}$ loop correspond to the residues that coordinate a $\mathrm{Ca}^{2+}$ ion $^{24}$ (Supplementary Fig. 5a, c). The D loop interacts with ECL2, which is connected to TM3 via the C481 ${ }^{3.29}-\mathrm{C} 573^{\mathrm{ECL} 2}$ disulfide bond (numberings of class A and class C GPCRs are based on $\mathrm{BW}^{28}$ and $\mathrm{Pin}^{29}$, respectively; Fig. 3a and Supplementary Fig. 5d).

In another subclass, we observed an apo GPR158 structure in which the CR linker is significantly compressed to 20 from $47 \AA$ (Supplementary Figs. $1 \mathrm{~g}$ and $5 \mathrm{e}$ ). Because the resolution of this region is not sufficient to build a model for this subclass structure, it is unclear which part is responsible for the reduced length of the CR linker. Conformational change of the GPR158 CR linker suggests that the region between the PAS and TM domains undergoes dynamic movement, implicating a role for this linker in communication between the two domains.

Comparison of the GPR158 TM domain with other class C GPCRs. The TM domains of the two protomers are virtually identical with an r.m.s.d. of $0.5 \AA$. The GPR158 TM domain is most similar to inactive GBR2 $(1.8 \AA)$ and most distant from active mGluR5 (3.1 $\AA$ ) in class C GPCRs ${ }^{30-37}$ (Supplementary Fig. 6a). Compared with other class C GPCRs, GPR158 TM4 is longer by one and a half turns at the extracellular end (Supplementary Fig. 6b). The most notable feature of a TM domain is extensive ionic networks present in three layers (Fig. 3a, b). While ion pairs in layers I and II are unique in GPR158, the ionic lock in layer III is conserved in other GPCRs. Layer I located below ECL2 contains an R485 3.33-D5795.40-R6316.57 network, to which Y $647^{7.32}$ makes an H-bond contact (Fig. 3b). Beneath this layer, E586 5.47 engages in ionic contact with $\mathrm{R} 488^{3.36}$ and H6286.54, which are buttressed by a polar network formed by $\mathrm{Y} 496^{3.44}$, 

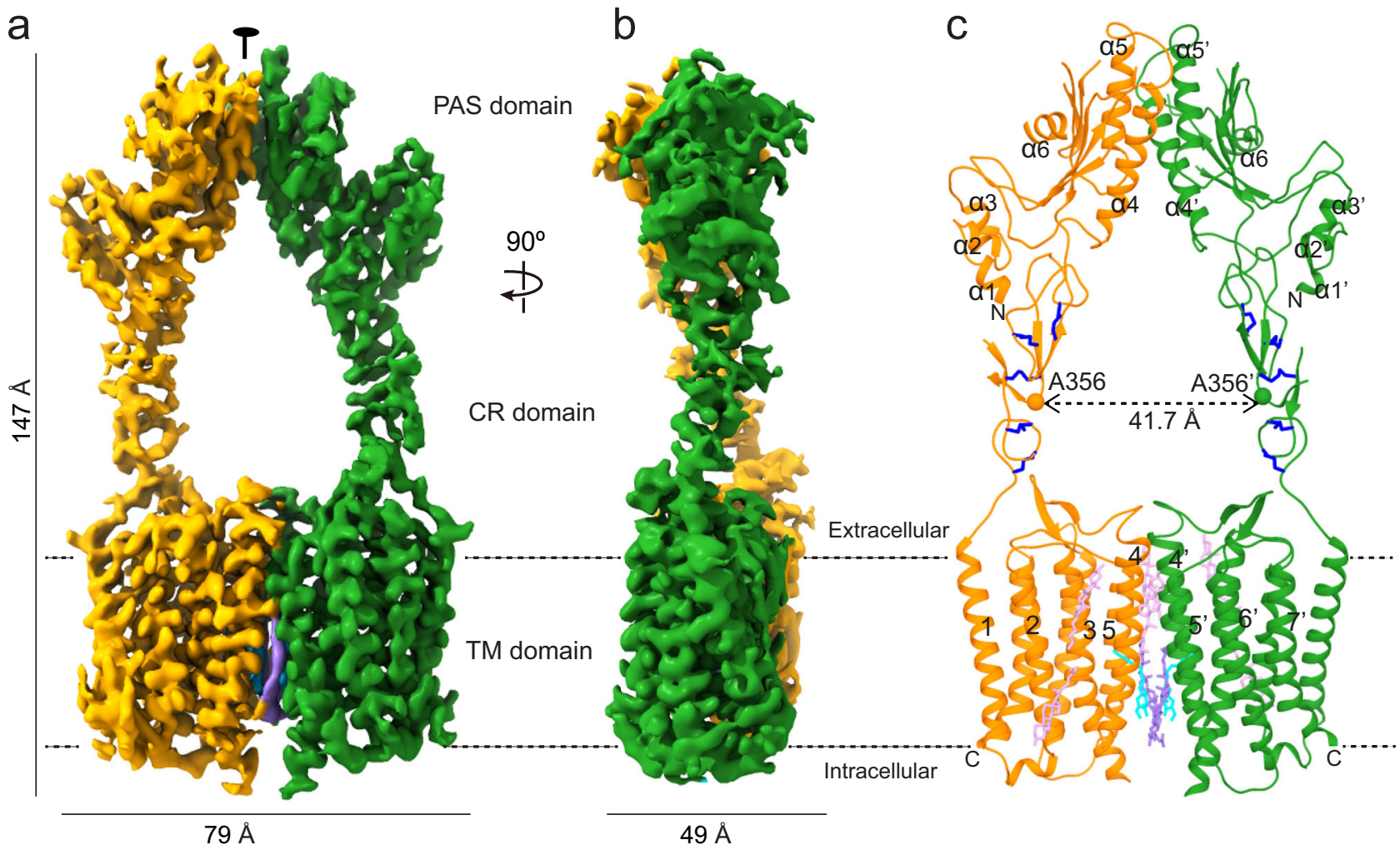

Fig. 1 Cryo-EM structure of GPR158. a, b Cryo-EM maps of the GPR158 homodimer in orthogonal views. c Model of GPR158 colored the same as in a. CHS, OG, and cholesterol molecules are represented as purple, sky blue, and pink sticks, respectively. Disulfide bonds are shown as blue sticks.
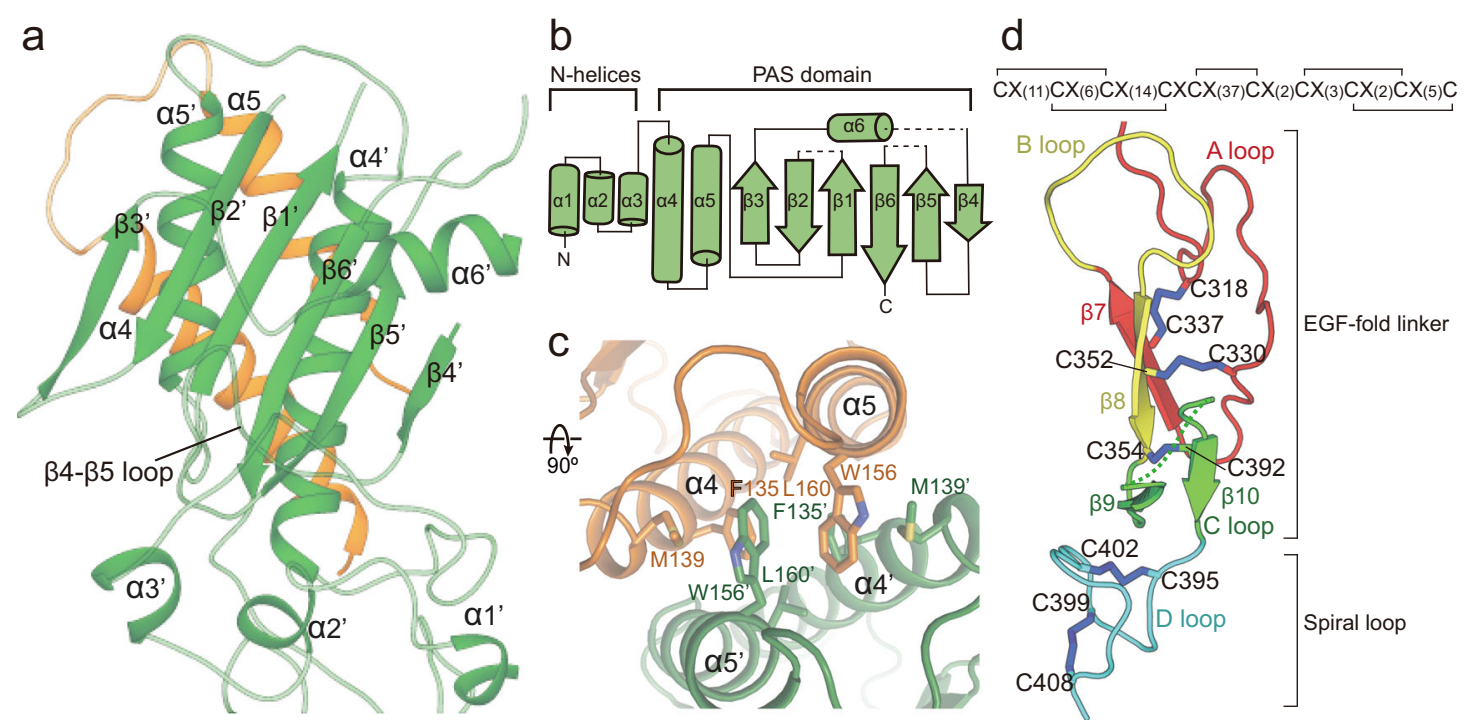

Fig. 2 Structure of the extracellular domain of GPR158. a Structure of the N-terminal helices (bottom) and the PAS domain (top) of GPR158 in the view shown in Fig. 1b. b Topology diagram of the N-terminal helices and the PAS core of GPR158. Dotted lines indicate disordered loops. c Close-up view of the dimeric interface between the GPR158 PAS domains in a top view from a. d The CR domain stabilized by disulfide bonds shown in black lines (top) and blue sticks (bottom).

$\mathrm{E} 620^{6.46}, \mathrm{~S} 624^{6.50}, \mathrm{H} 650^{7.35}$, and $\mathrm{T} 654^{7.37}$. Layer III contains an ionic lock, a hallmark of the inactive state in other GPCRs, by tethering TM3, TM6, and ICL3. In this ionic network, the K666 $66^{7.51}$-E609ICL3 $-K 502^{3.50}$ cluster is positioned near the cytoplasmic end. K502 3.50 also forms an $\mathrm{H}$-bond with S450ICL1. K666 6.51 from the LxPKxx motif is equivalent to FxPKxx in TM7 in other class C GPCRs and NPxxY in class A GPCRs ${ }^{31}$
(Supplementary Fig. 7a). K502 3.50 is equivalent to $\mathrm{K}^{3.50}$ that interacts with D688 in the D/ERY motif in ICL3 in the $\mathrm{GABA}_{\mathrm{B}}{ }^{31-35}$. Overall, the extensive ionic and polar networks in the three layers stabilize TM3, TM5, TM6, TM7, ICL1, and ICL3 in apo GPR158 (Fig. 3c). The polar networks of the top two layers occupy the orthosteric or allosteric binding sites in other GPCRs $^{38-43}$ (Fig. 3d, e). As a result, the space inside the GPR158 

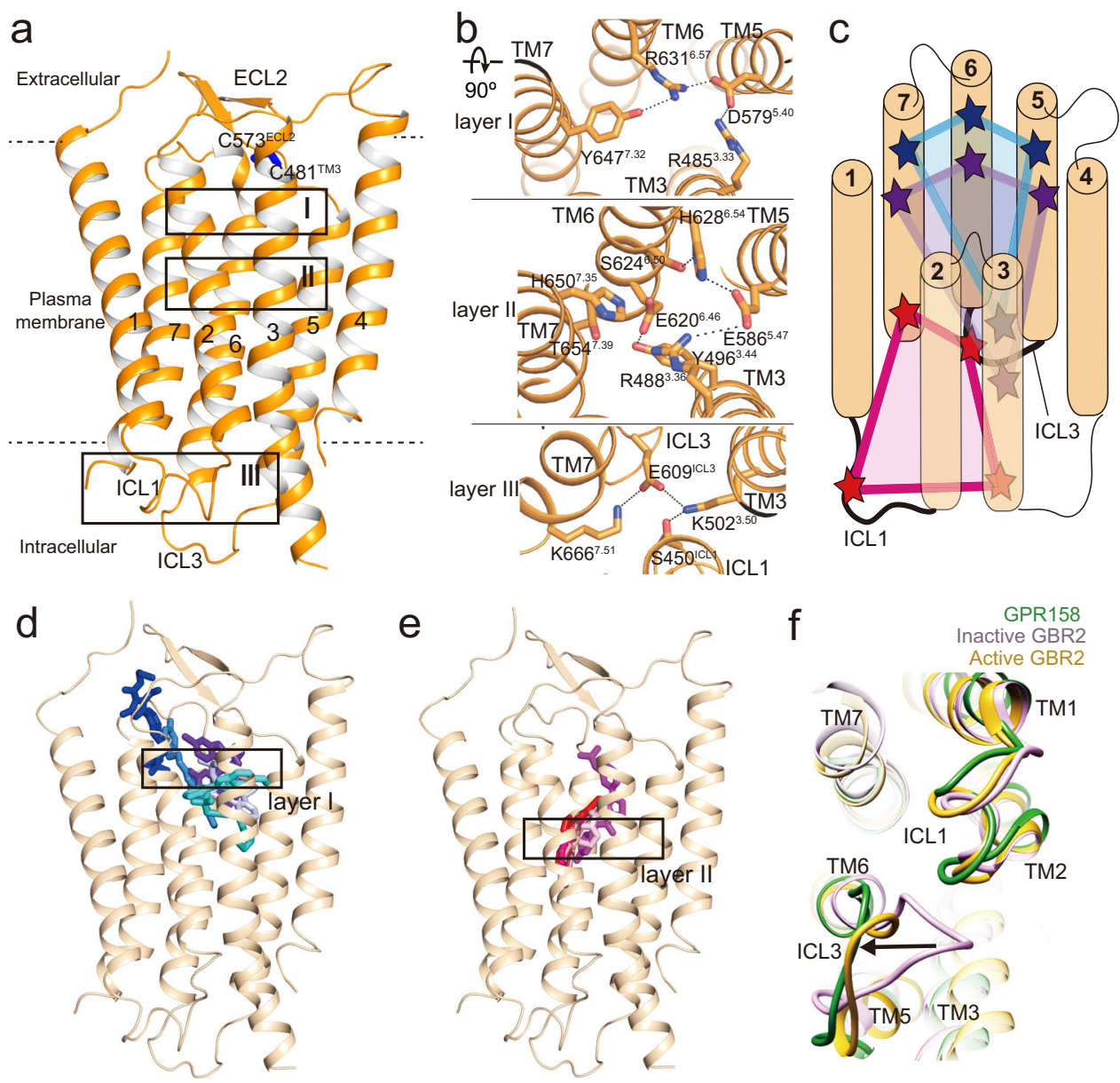

Fig. 3 Structure of the TM domain in comparison with other GPCRs. a Overall structure of the 7TM domain of GPR158. The opposite protomer is omitted for clarity. Boxes include clusters of interactions within a TM domain. The disulfide bond between ECL2 and TM3 are colored blue. $\mathbf{b}$ Close-up views of the three layers of ion pairs within a TM domain in an orthogonal view from a. The ionic and polar interaction network in layer I (top) and II (middle) stabilize $\mathrm{TM} 3,5,6$, and 7. c Cartoon representation of intra-TM domain interactions formed by layer I (blue), II (purple), and III (pink). d, e Location of the orthosteric ligands bound to class A (d) and the allosteric ligands bound to class C (e) GPCRs in comparison with layer I and II within the GPR158 TM domain (light orange). The ligands are shown as sticks and colored as follows: GPR52 (6LIO, blue), MT1 (6ME3, cyan), mAChR (6OIJ, purple blue), A2A (6SOL, sky blue), OX1R (6V9S, purple), and ADRB2 (6OBA, light blue) from class A (d), and mGluR1 (4OR2, magenta) and mGluR5 (4OO9, light pink; $5 C G C$, red; $5 C G D$, raspberry; $6 \mathrm{FFH}$, salmon; $6 \mathrm{FFI}$, hot pick) from class $\mathrm{C}(\mathbf{e})$. $\mathbf{f}$ Comparison of TM domain structures between GPR158 and inactive or active $\mathrm{GABA}_{\mathrm{B}}$ by aligning TM domains of GPR158 with the corresponding GBR2 structures. Conformational differences in ICLs between GPR158 and inactive GBR2 are indicated by an arrow.

TM domain is not sufficient for the ligand binding. Two cavities within the GPR158 TM domain are estimated to be 286 and $464 \AA^{3}$, significantly smaller than those in other class C or class A GPCRs, which range from 833 to $2832 \AA^{38-43}$ (Supplementary Fig. 6c).

Another notable feature of the GPR158 TM domain is the ICL1 and ICL3 conformation. In GPR158, ICL1 and ICL3 are straight and directed away from TM3 and TM6, resulting in open conformations similar to those in the active state of $\mathrm{GABA}_{\mathrm{B}}{ }^{32-34}$ (Fig. 3f). Thus, the structure of the GPR158 TM domains exhibit structural features of both inactive and active states of class C GPCRs.

Dimeric arrangement of the TM domains. In apo GPR158, the TM4/5 helices are arranged in an inverted V-shape at the dimeric interface (Figs. 1c and 4a). The residues at the top half of TM4 and TM5 near the extracellular end make direct contacts each other, whereas the bottom half at the intracellular end interact only through lipid molecules (Fig. 4a). The top half of the interface buries $1623 \AA^{2}$ of the surface area. Near the extracellular end, W5394.45 and F540 4.46 are packed against W578 5.39 , and the hydrophobic cluster interacts with $\mathrm{M} 581^{\prime 5.42}$, W578 $8^{\prime 5.39}$, and F540'4.46 of an opposite protomer (Fig. 4b). The other side of W578 5.39 packs against W539/4.45 and buttresses the interactions described above. W5394.45 is replaced by Gly in GPR179, whereas W578 5.39 and F540 4.46 are conserved (Supplementary Fig. 4c). Assuming that GPR179 forms a similar dimer, the structure suggests that the W5785.39-F540 4.46 contact is critical for the formation of a dimeric interface (Fig. 4b). In GBR1, GBR2, and mGluR5, F540 4.46 is replaced by Thr or Ile, whereas W578 5.39 is conserved or replaced by Gly, explaining why the formation of the TM4/5 interface is favorable in GPR158 (Supplementary Fig. 7a). At the bottom half of the TM interface, we observed welldefined but unknown densities and assigned them as putative octyl- $\beta$-D-glucopyranoside (OG) and cholesterol hemisuccinate (CHS), which were added during sample preparation (Fig. 4a, b and Supplementary Fig. 2g).

The inverted V-shaped arrangement of the TM4/5 helices at the GPR158 interface is in marked contrast to other class C 

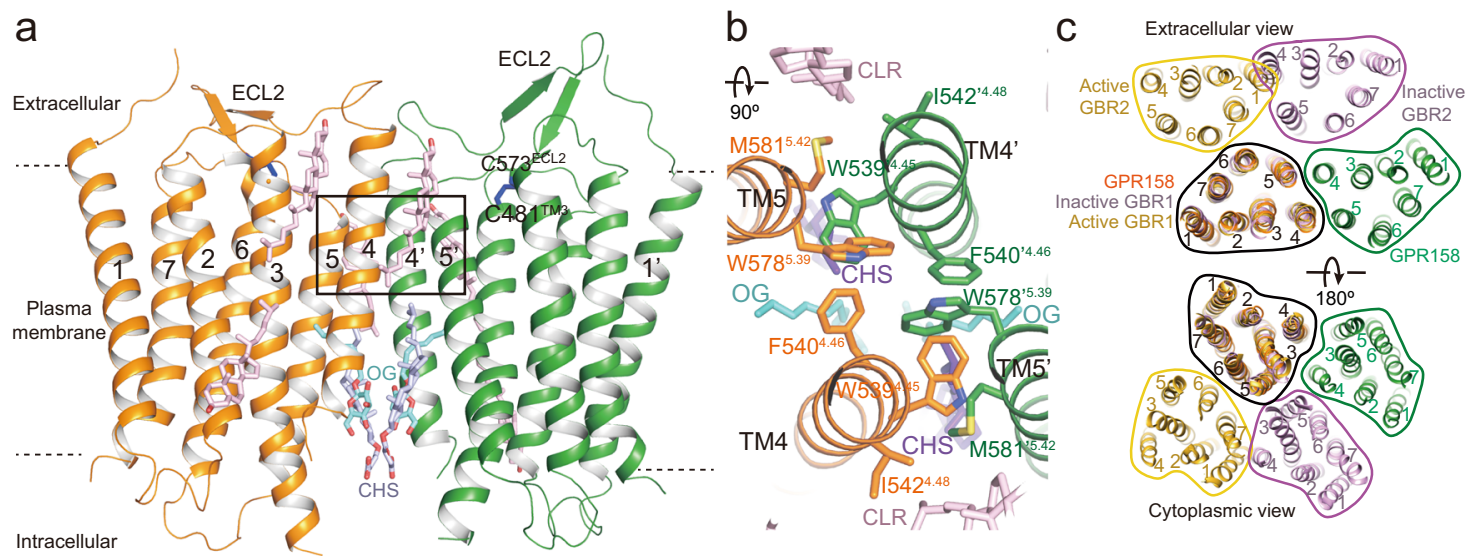

Fig. 4 Dimeric arrangement of the TM domains. a Overall structure of the 7TM domains of the GPR158 homodimer. CHS, OG, and cholesterol molecules are represented as purple, sky blue, and pink sticks, respectively. A box includes clusters of interactions at the dimeric interface of the TM domains. The disulfide bonds between ECL2 and TM3 are colored blue. $\mathbf{b}$ The TM4/5 dimeric interface looking down from top of $\mathbf{a}$. c Comparison of the dimeric interface

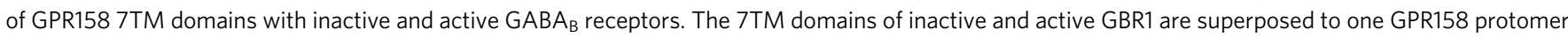
(black line). Both extracellular (top) and cytoplasmic (bottom) views are shown.

GPCRs ${ }^{30-37}$. Upon superposition of the first TM domains of class C GPCRs, the second TM domain of GPR158 is rotated by $90^{\circ}$ with respect to the second $\mathrm{TM}$ domain of inactive $\mathrm{GABA}_{\mathrm{B}}$ and mGluR5 in a counterclockwise direction, and rotated by $60^{\circ}$ relative to that of CaSR (Fig. 4c and Supplementary Fig. 6d). Similar to apo GPR158, TM helices at the interface in active $\mathrm{GABA}_{\mathrm{B}}$ form an inverted V-shape. However, TM6/7 rather than $\mathrm{TM} 4 / 5$ are arranged at the dimeric interface. Because all class $\mathrm{C}$ GPCRs involve TM6-TM6 interface formation in the active state, we hypothesized that GPR158 might adopt a similar conformation upon activation; hence, we modeled a putative GPR158 dimer by aligning each GPR158 TM domain onto GBR1 and GBR2 of active GABA $\mathrm{B}_{\mathrm{B}}$ (Supplementary Fig. 6e). Although several hydrophobic residues pack against each other, positively charged residues (Arg637 at the extracellular side; Arg601, H608, and Arg611 at the cytoplasmic side) face each other at the interface, resulting in repulsion. This suggests that the GPR158 TM domains are unlikely to form a TM6-TM6 ${ }^{\prime}$ interface in their current conformations.

Structures of the GPR158-RGS7-G $\boldsymbol{\beta} 5$ complex. The GPR158 TM domain exhibits both inactive and active features of class $C$ GPCRs; hence, we cannot elucidate whether apo GPR158 is in an inactive, partially active or fully active state that can directly

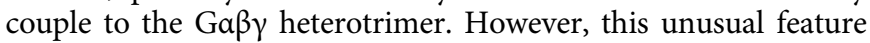
might be explained by the noncanonical signaling mechanism of GPR158, in which the apo GPR158 receptor recruits and inactivates the Gai/o protein via the RGS7-G 35 heterodimer ${ }^{7,8}$. The apo GPR158 recruits the RGS7-G $\beta 5$ heterodimer and Gai/o protein via the first- and second half of the cytoplasmic domain, respectively ${ }^{7}$. Cryo-EM analyses for GPR158 (residues 1-863)-RGS7-G $\beta 5$ complex revealed three types of GPR158 states: apo GPR158 and the GPR158:RGS7:G $\beta 5$ complex in a 2:1:1 ratio at an average resolution of $4.3 \AA$ and in a 2:2:2 ratio at $4.7 \AA$ (Fig. 5a, Supplementary Figs. 8 and 9 and Supplementary Table 1). In both complexes, structures of GPR158 and the four domains of RGS7 DEP (Dishevelled, Egl10, Pleckstrin), DHEX (DEP helical extension), GGL linker, and the RGS domain, and the G $\beta 5$ subunit were clearly visible and their models fitted well into the cryo-EM maps (Supplementary Fig. 9o, p). However, most of the cytoplasmic domain of GPR158 (residues 669 to 863) is disordered except for a coiled-coil (Ha and $\mathrm{Hb}$ ) comprising 61 residues (Supplementary Figs. 4d and 9s).
In this coiled-coil, the first helix (Ha) forms seven turns from 27 residues, and the second helix $(\mathrm{Hb})$ of 22 residues forms six turns, and they are connected by an eight-residue linker (Supplementary Figs. 4d and 9s). Although we could not assign the side chains of the cytoplasmic helices, biochemical studies and secondary structure prediction suggest that this region is likely to correspond to residues 708 to 763 (refs. ${ }^{7,8}$ ). The two helices are rich in Leu and Ile, suggesting that they form a coiled-coil through these residues, consistent with the three-dimensional (3D) model predicted using the I-TASSER program ${ }^{44}$ (Supplementary Fig. 9t).

Since the 2GPR158-RGS7-G $\beta 5$ and 2GPR158-2RGS7-2G $\beta 5$ complexes exhibit virtually identical structures, except for an additional RGS7-G $\beta 5$ heterodimer in the latter, we focus on describing the overall structure of the 2GPR158-2RGS7-2G 35 complex and close-up view of the 2GPR158-RGS7-G $\beta 5$ complex (Fig. 5a and Supplementary Fig. 10a). The RGS7-G 35 heterodimer binds to GPR158 through the DHEX domain, which is sandwiched between the ICLs and the Ha helix (Fig. 5a and Supplementary Fig. 10a, b). The disordered ICL2 (10 residues) and the cytoplasmic tip of TM3 in apo GPR158 becomes ordered upon binding of RGS7 (Fig. 5b and Supplementary Fig. 9q). Conformation of the three ICLs of GPR158 bound to the RGS7 are similar to those of Gi-bound $\mathrm{GABA}_{\mathrm{B}}, \mathrm{mGluR2}$, and mGluR434,36,45,46 (Fig. 5c). The RGS7-GB5 complex is tilted $\sim 40^{\circ}$ relative to the plasma membrane in a way that both the DEP and DHEX domains are located closest to the TM domains, and the RGS domain is farthest (Fig. 5a and Supplementary Fig. 10a). The DHEX domain faces the ICLs, inserting the Xal helix and the Xa1Xa2 loop to the inverted V-shaped TM interface (Fig. 5b). This loop is also involved in binding to $\mathrm{R}^{\mathrm{BP}}{ }^{47}$. The $\mathrm{Xa} 1$ and $\mathrm{Xa} 3$ helices of the DHEX domain are packed below the GPR158 TM (ICL2, ICL3, and TM3), and the Xa3 helix is on top of the Ha helix that is positioned in perpendicular to the TM helices (Fig. 5a, d and Supplementary Fig. 10a, b, c). The linker between the end of TM7 and the Ha helix is disordered and the distance between the two ends of the disordered region is $47 \AA$ in one protomer and $53 \AA$ in the other. Thus, it is unclear to which protomer the coiled-coil belongs. The GPR158 fragment (residues 665-775) containing the coiled-coil is sufficient for binding to the RGS7-G 35 complex, illustrating the importance of the Ha helix in binding to RGS7 (ref. ${ }^{7}$ ). Superposition of the RGS7-G $\beta 5$ dimer in the complex onto the reported structure of free 

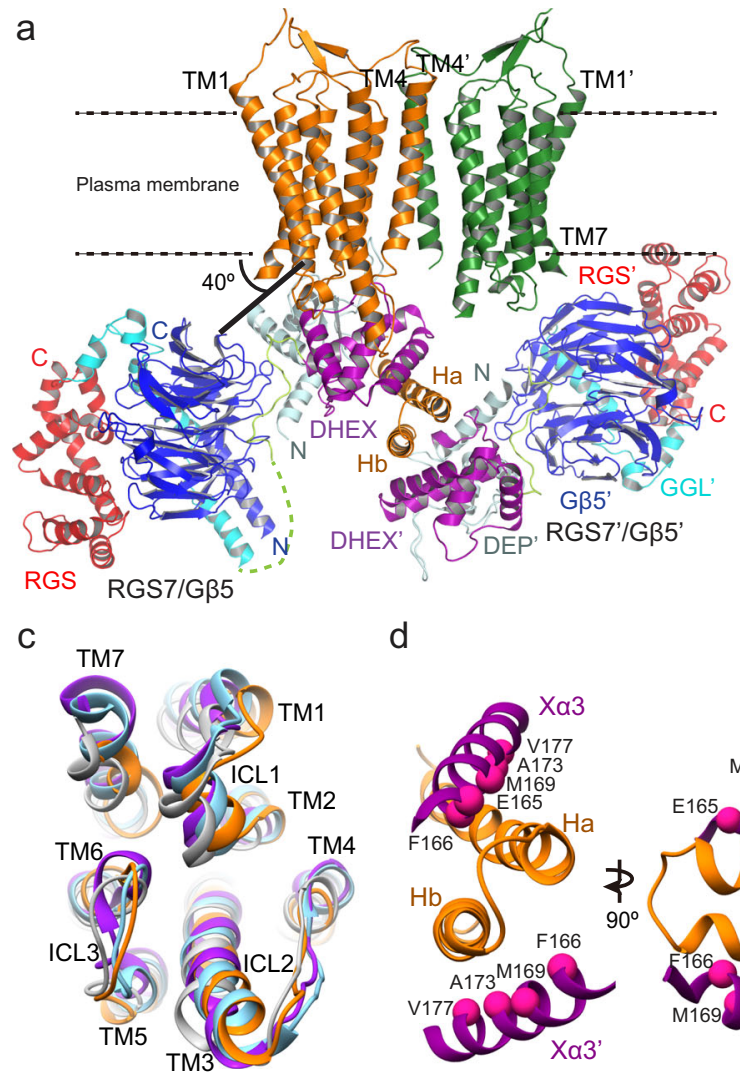

d

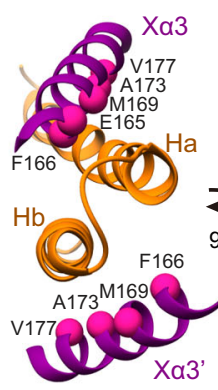

b

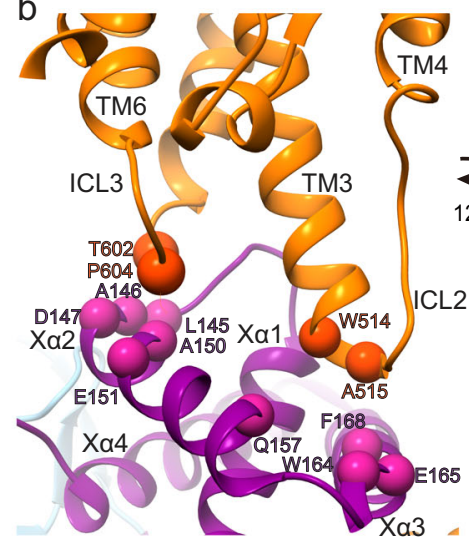

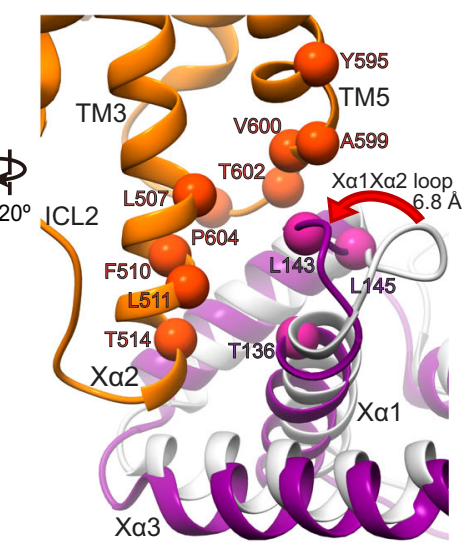

e

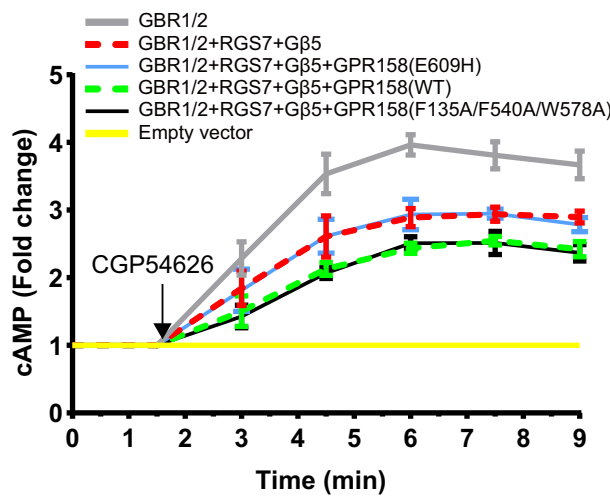

Fig. 5 Cryo-EM structures of the GPR158-RGS7-G $\beta 5$ complex. a Overall structure of the 2GPR158-2RGS7-2G $\beta 5$ complex. Each domain is colored as follows: each 7TM domain of GPR158, orange and green, respectively; DEP, light cyan; DHEX, purple; GGL-RGS linker, light green; GGL, cyan; RGS, red; G $\beta 5$, blue. The disordered region between DHEX and GGL domains is indicated by a light green dotted line. $\mathbf{b}$ Close-up view of the interaction between the DHEX domain and GPR158. Residues at the interface are shown as spheres. The $120^{\circ}$-rotated view (right) highlights the conformation change of the X $\alpha 1$ X 22 loop upon GPR158 binding. The RGS7 bound to GPR158 is aligned with the RGS7-G 35 dimer 47 (PDB 6N9G, light gray). c Superimposed 7TM domain of GPR158 bound to RGS7-G $\beta 5$ (orange) with each 7TM domain of the GABA - Gi (PDB 7EB2, light gray), mGluR2-Gi (PDB 7MTS, purple), and mGluR4-Gi (PDB 7E9H, sky blue) viewed from the bottom. $\mathbf{d}$ Interactions between X $\alpha 3$ and the cytoplasmic helices in two views. e CGP54626-induced CAMP production using HEK293T cells expressing indicated GPR158 mutants. Data represent mean values with standard deviations from three independent experiments.

RGS7-G $\beta 5$ showed that the two structures are almost identical with an r.m.s.d. of $1.3 \AA^{47}$. However, the Xa1Xa2 loop of DHEX is shifted towards TM5 by as much as $6.8 \AA$, consistent with previous reports that the loop is the most flexible part of the RGS7 protein ${ }^{47}$ (Fig. 5b).

To understand if the TM domain arrangement of the GPR158 dimer is important for binding of RGS7-G $\beta 5$, we aligned a TM domain of the GPR158-RGS7-G 35 complex to a TM domain of inactive or active CaSR, mGluR5, and $\mathrm{GABA}_{\mathrm{B}}$ receptors $^{30-35}$ (Supplementary Fig. 11a, b). In all cases, RGS7-G $\beta 5$ collides to TM domain of the other protomer. This suggests that the inverted V-shaped TM4/5 arrangement of apo GPR158 is critical for recruiting RGS7-G $\beta 5$, and explains why apo GPR158 can facilitate noncanonical signaling.

Another RGS7-G $\beta 5$ heterodimer binds to the Hb helix, which is visible only in one GPR158 protomer (Fig. 5a, d and Supplementary Fig. 10d). The second RGS7-G 35 dimer is oriented in pseudo-twofold symmetry with respect to the first dimer against the Ha helical axes (Fig. 5a). Similar to the first dimer, binding of the second RGS7-G $\beta 5$ complex is achieved through helix $\mathrm{Xa3}^{\prime}$ from the DHEX' domain (Fig. 5d and Supplementary Fig. 10d). While DHEX' of the second RGS7 binds to the Hb helix, the RGS' domain of RGS7' shifts proximal to the micelles (Supplementary Movie 1). Thus, although the interaction between the second RGS7 and $\mathrm{Hb}$ helix is not as extensive as the interaction of the first RGS7, the RGS7'-micelle interaction contributes to the stability of the second RGS7-G $\beta 5$ in the GPR158-RGS7 complex.

Apo GPR158 not only provides a platform for the binding of RGS7 and Gai/o activated by other GPCRs such as $\mathrm{GABA}_{\mathrm{B}}$, but also potentiates GTP hydrolysis, thereby terminates both Ga and $G \beta \gamma$ signaling pathways ${ }^{7,8,11}$. To examine if the GPR158 structure affects the cAMP production, we engineered two different classes of GPR158 mutants in which the dimeric interface or intra-TM ion pairs were disrupted, and examined their cAMP-producing activity (Fig. 5e and Supplementary Fig. 12). Overexpression of the RGS7-G 35 complex alone decreased the amount of cAMP produced by adenylate cyclase as previously reported ${ }^{11}$. Coexpression of GPR158 and RGS7-G $\beta 5$ further reduced the cAMP production. For the first class mutant, we simultaneously replaced the residues at the extracellular (F135A) and TM (F540A, W578A) domains (Figs. $2 \mathrm{c}$ and $4 \mathrm{~b}$ ). The dimer-disrupting mutant exhibited similar cAMP-producing activity relative to wild-type GPR158 (Fig. 5e and Supplementary Fig. 12a). Next, we mutated the intra-TM ion pairs in layer III (E609H) (Fig. 3b). Cells expressing the GPR158 E609H mutant exhibited increased cAMP 
production compared with those expressing wild-type GPR158, similar to those lacking GPR158 (Fig. 5e and Supplementary Fig. 12a). This result suggests that a GPR158 protomer is sufficient for binding of the RGS7-G 35 heterodimer and Gai/o protein and potentiating GTP hydrolysis activity by RGS7. However, TM domain structure is important for the activation of noncanonical signaling.

\section{Discussion}

The PAS domain functions as a molecular sensor in various signaling pathways in all kingdoms of life ${ }^{21,48}$. Because of its general role in recognizing diverse ligands and proteins, the PAS domain has been observed in various signaling proteins including histidine kinases, nucleotide cyclases and response regulators ${ }^{48}$. In this work, we extend diversity of the PAS domain and present the first example of 7TM fused to the PAS domain in GPCRs. Because GPR158 and GPR179 are homologous, GPR179 is expected to adopt a similar overall structure. Structural similarity of the GPR158 extracellular core with those of chemotaxis receptor and histidine kinases suggests that GPR158 might interact with ligands through a conserved binding-pocket ${ }^{22,23}$ (Supplementary Fig 3a, c). Alternatively, GPR158 might interact with the PAS-associating proteins ${ }^{49}$.

The TM domains of apo GPR158 adopt a conformation reflecting both active and inactive states of other class C GPCRs. Furthermore, the TM4/5 interface is a unique feature of apo GRP158. Although these features are complicated to explain the activation of $\mathrm{G}$ protein via agonist-dependent direct coupling, the structure provides a clue for the RGS7-G 35 binding. Open conformation of ICL3 that resembles active GBR2 contributes to the interaction with the DHEX domain of RGS7 (Figs. $3 \mathrm{f}$ and $5 \mathrm{~b}$ ). The RGS7-G $\beta 5$ heterodimer cannot be localized to the TM domains in inactive or active state of other class C GPCRs, which suggests that only the TM dimer with the TM4/5 interface in a GPR158 geometry can recruit the RGS7 complex (Supplementary Fig. 11a, b). Although a GPR158 monomer can recruit the RGS7 complex and facilitate GTP hydrolysis, it is possible that the ligand binding further accelerates GTPase activity in GPR158 dimer as previously shown in melatonin $\mathrm{MT}_{1}$ receptor ${ }^{50}$. Furthermore, dimerization may be important in canonical signaling, in which binding of an agonist to a dimer directly activates $G$ protein.

Termination of signaling is a highly sensitive and critical event, which can be controlled by the specific interactions between RGS proteins and subsets of Ga proteins as well as between GPCRs and RGS proteins ${ }^{12,51,52}$. Although relatively few examples of GPCR and RGS interactions have been identified, several GPCRs directly and selectively recruit various RGS proteins ${ }^{12}$. In particular, among class C GPCRs, GPR158 is unique in that it only interacts with RGS7 and RGS6 in the RGS family and recruits them to the plasma membrane in order to regulate $G$ protein activity $^{1}$. The GPR158-RGS7-G 35 structure provide a basis for the selectivity of GPR158 for RGS proteins: first, only RGS7 family members contain the DHEX domain, which is responsible for binding GPR158. In many RGS proteins, the DEP domain has been reported to interact with GPCRs ${ }^{53,54}$. Second, the relative orientation of DEP and DHEX domains in RGS7 and RGS9 differs substantially 47,55 . Furthermore, the helices in the DHEX domain are organized differently in these RGS proteins ${ }^{47}$. Aligning RGS9 with RGS7 in the GPR158-RGS7-Gß5 complex structure results in steric collision between RGS9 and GPR158 (Supplementary Fig. 10e). Third, the Xa1X $\alpha 2$ loop, a key element in recognizing GPR158, differs significantly from the RGS9 loop (Supplementary Fig. S10e). Furthermore, RGS6 and RGS7 share highly conserved sequence of the Xa1Xa2 loop, whereas RGS9 and RGS11 exhibited clear differences (Supplementary Fig. 7b). The conformation of the Xa1Xa2 loop changes upon binding to GPR158 (Fig. 5b). Since this loop is also involved in R7BP binding 47 , the Xa1Xa2 loop might be an important mediator of RGS7 in binding to other GPCRs. Fourth, the GPR158-contacting residues in the DHEX domain are highly conserved in RGS6, but not in RGS9 and RGS11 (Supplementary Fig. 7b). Conversely, the cytoplasmic coiled-coil of GPR158 is crucial for recruiting the DHEX domain of RGS7 ( ref. $^{7}$ ). The cytoplasmic coiled-coil and the DHEX-contacting residues are highly conserved in GPR179, but not in other GPCRs (Supplementary Fig. 4d). These data suggest that GPR158 and GPR179 bind RGS7 protein in a similar manner. Furthermore, because the RGS7-binding region of GPR158 shares similarity with R7BP and R9AP, it is possible that the binding mode of R7BP and R9AP proteins for RGS7 is similar to that of GPR158.

GPCRs are known to interact with various RGS proteins via their ICL3 or C-terminal tail ${ }^{12}$. The GPR158-RGS7 interaction is much more extensive than other reported GPCR-RGS interactions. The GPR158-RGS7-G 35 complex structures suggest that direct coupling of GPR158 with $G$ protein via ICLs similar to those observed in $\mathrm{GABA}_{\mathrm{B}}$, mGluR2, mGluR4, and Ste2 is unlikely in the presence of RGS7-G $\beta 5$ complex because the RGS7-G $\beta 5$ complex and $G$ protein would collide each other $36,45,46,56$ (Supplementary Fig. 11c). Instead, GPR158 recruits the Gai/o subunit in various states through the first half of the cytoplasmic domain and places the RGS7 and Gai/o proteins in close proximity. Because the ligand-free GPR158 can stimulate GTPase activity, it would be reasonable to speculate that apo GPR158 triggers rearrangement of RGS7 and Gi proteins to interact each other. Further structural analysis is required to determine how binding of $\mathrm{Ga}$ to the cytoplasmic half activates RGS7. It is unclear if the apo GPR158's activity that potentiates GTP hydrolysis is partial or full. However, stimulation of GTP hydrolysis by the ligand-free GPR158 is critical because GPR158-RGS7-G $\beta 5$ complex turns off the Gi/o signaling activated by other GPCRs and regulates ion channels such as $G$ protein-coupled inwardlyrectifying potassium and voltage-gated calcium channels ${ }^{10,11}$. Finally, structural information of the GPR158 PAS domain and the GPR158-RGS7 interface can provide an attractive framework to design antidepressant drugs ${ }^{3}$.

\section{Methods}

Expression and purification of apo GPR158. The gene encoding human GPR158 was purchased from Addgene (\#66332), amplified by PCR, and cloned into the pEG BacMam vector using restriction enzymes EcoRI and $N o t \mathrm{I}^{57}$. The resulting GPR158 constructs encoding amino acid residues $1-710$ or residues $1-863$ were fused with a PreScission protease cleavage site, green fluorescent protein (GFP), and a Twin-Strep II tag at the C-terminal end. Baculoviruses harboring GPR158 were generated in Spodoptera frugiperda (Sf9) cells using the Bac-to-Bac system (Invitrogen). For expression of apo GPR158, HEK293S $\mathrm{GnTI}^{-}$cells grown to a density of $3.0 \times 10^{6}$ cells $/ \mathrm{ml}$ were infected with recombinant baculoviruses and cultured for $12 \mathrm{~h}$ at $37^{\circ} \mathrm{C}$. Sodium butyrate $(10 \mathrm{mM})$ was added, and cells were further incubated for $48 \mathrm{~h}$ at $30^{\circ} \mathrm{C}$.

Cells were collected by centrifugation and resuspended in lysis buffer containing $20 \mathrm{mM}$ HEPES pH 7.5, $300 \mathrm{mM} \mathrm{NaCl}$, and $1 \mathrm{mM}$ EDTA. Cell membranes were solubilized with $20 \mathrm{mM}$ HEPES pH $7.5,300 \mathrm{mM} \mathrm{NaCl}, 1 \%$ (w/v) lauryl maltose neopentyl glycol (LMNG; Anatrace), 0.1\% (w/v) CHS; Sigma), 20\% glycerol, $1 \mathrm{mM}$ EDTA, $1 \mathrm{mg} / \mathrm{ml}$ iodoacetamide, $150 \mu \mathrm{g} / \mathrm{ml}$ benzamidine, and protease inhibitor cocktail (Roche) for $1 \mathrm{~h}$, and then cleared by ultracentrifugation at $125,171 \times g$ with a $45 \mathrm{Ti}$ rotor (Beckman) for $1 \mathrm{~h}$ at $4{ }^{\circ} \mathrm{C}$. The supernatant was isolated and applied to StrepTactin XT resin (IBA Lifesciences) for $3 \mathrm{~h}$. The resin was thoroughly washed with $20 \mathrm{mM}$ HEPES pH 7.5, $300 \mathrm{mM} \mathrm{NaCl}, 0.1 \%$ LMNG, $0.01 \%$ CHS, $10 \%$ glycerol, and $1 \mathrm{mM}$ EDTA. To elute the resin-bound protein, PreScission protease was added at a ratio of 10:1 (w/w) and incubated overnight in buffer comprising $20 \mathrm{mM}$ HEPES pH 7.5, $200 \mathrm{mM} \mathrm{NaCl}, 0.01 \%$ LMNG, $0.001 \%$ CHS, $10 \%$ glycerol, and $5 \mathrm{mM}$ EDTA. The resin was transferred to a gravity column, and the flowthrough fraction containing GFP-cleaved GPR158 was collected. The protein was concentrated to $6.9 \mathrm{mg} / \mathrm{ml}$ using an Amicon Ultra centrifugal device (100 kDa cutoff; Millipore) and stored at $-80^{\circ} \mathrm{C}$. 


\section{Expression and purification of the GPR158-RGS7-G $\beta 5$ complex. Genes} encoding human RGS7 (\#55760) and GB5 (\#55763) were purchased from Addgene and cloned into $\mathrm{pEG}$ BacMam vectors to include a Flag tag fused at the C-terminal end of RGS7. Expression of the GPR158-RGS7-G 35 complex was induced as described above for apo GPR158, except that three kinds of baculoviruses each harboring GPR158, RGS7, or G $\beta 5$ were used at a ratio of 1:1:1. Cultured cells were collected by centrifugation at $4647 \times g$ for $30 \mathrm{~min}$, and lysed in buffer containing $20 \mathrm{mM}$ HEPES pH 7.5, $500 \mathrm{mM} \mathrm{NaCl}$, and $1 \mathrm{mM}$ EDTA. Cells were disrupted on ice using a Dounce homogenizer (Kimble) and solubilized in buffer comprising $20 \mathrm{mM}$ HEPES pH 7.5, $500 \mathrm{mM} \mathrm{NaCl}, 1 \mathrm{mM}$ EDTA, 1\% LMNG, 0.1\% CHS, 20\% glycerol, $1 \mathrm{mg} / \mathrm{ml}$ iodoacetamide, and protease inhibitor cocktail (Roche) for $1 \mathrm{~h}$ at $4{ }^{\circ} \mathrm{C}$. The solubilized membranes were isolated by ultracentrifugation with a Ti45 rotor (Beckman) at $138,001 \times g$ for $1 \mathrm{~h}$ at $4^{\circ} \mathrm{C}$, followed by incubation with antiFlag affinity G1 resin (GenScript) for $1 \mathrm{~h}$ at $4^{\circ} \mathrm{C}$. The resin was washed in batch with $20 \mathrm{mM}$ HEPES pH 7.5, $300 \mathrm{mM} \mathrm{NaCl}, 10 \%$ glycerol, $1 \mathrm{mM}$ EDTA, $0.1 \%$ LMNG and $0.01 \%$ CHS. The resin was then transferred to an EconoPac column and further washed with $20 \mathrm{mM}$ HEPES pH 7.5, $200 \mathrm{mM} \mathrm{NaCl}, 10 \%$ glycerol, $1 \mathrm{mM}$ EDTA, $0.01 \%$ LMNG, and $0.001 \%$ CHS. Proteins were eluted using $20 \mathrm{mM}$ HEPES pH 7.5, $200 \mathrm{mM} \mathrm{NaCl}, 10 \%$ glycerol, 5 mM EDTA, 0.01\% LMNG, $0.001 \%$ CHS, and $0.4 \mathrm{mg} / \mathrm{ml}$ Flag peptide. Eluted proteins were concentrated using an Amicon Ultracentrifugal device ( $100 \mathrm{kDa}$ cut-off; Millipore) and injected onto a Superose 6 10/300 column equilibrated with buffer comprising $20 \mathrm{mM} \mathrm{HEPES} \mathrm{pH}$ 7.5, $200 \mathrm{mM} \mathrm{NaCl}, 1.5 \%$ glycerol, $5 \mathrm{mM}$ EDTA, $0.01 \%$ LMNG, and 0.001\% CHS. Eluted fractions containing GPR158, RGS7, and G $\beta 5$ were pooled and concentrated to $4.5 \mathrm{mg} / \mathrm{ml}$ using a Vivaspin device (100 kDa cut-off; GE Healthcare) for cryoEM analysis.

\section{Cryo-EM sample preparation and data collection for apo GPR158. Apo}

GPR158 was freshly prepared for cryo-EM grids immediately after size exclusion chromatography, which was performed using a Superose $610 / 300$ column (GE Healthcare) equilibrated with $20 \mathrm{mM}$ HEPES pH 7.5, $200 \mathrm{mM} \mathrm{NaCl}, 0.01 \%$ LMNG, $0.001 \% \mathrm{CHS}$, and $5 \mathrm{mM}$ EDTA. Peak fractions containing GPR158 were pooled and concentrated in a Vivaspin device (100 kDa cut-off; GE Healthcare). The concentrated protein was supplemented with $0.1 \%$ OG (Anatrace) and incubated for $1-1.5 \mathrm{~h}$ prior to vitrification. Samples $(3 \mu \mathrm{l})$ at a concentration of $12.7 \mathrm{mg} / \mathrm{ml}$ were applied to glow-discharged holey carbon grids (C-flat 1.2/1.3 Au 400-mesh grid; EMS). Grids were plunge-frozen in liquid ethane using a Vitrobot Mark IV (Thermo Fisher Scientific) with a blot force of 3 and blot time for $5 s$ at $100 \%$ humidity and $4{ }^{\circ} \mathrm{C}$.

Images were acquired using a Talos Arctica electron microscope (FEI) operated at $200 \mathrm{kV}$ and equipped with a Gatan K3 summit direct electron detector in counting mode (Photon Science Center at Pohang University of Science and Technology) at a nominal magnification of $\times 100,000$. Movies were collected comprising 10,361 micrographs. Datasets were collected with a pixel size of $0.83 \AA$ and a defocus of -0.6 to $-1.2 \mu \mathrm{m}$. Micrographs were dose-fractionated over 50 frames with a dose rate of 9 electrons per pixel per second and a total exposure time of 3.9 s, resulting in an accumulated dose of 50 electrons per $\AA^{2}$.

Cryo-EM sample preparation and data collection for the GPR158-RGS7-G $\beta 5$ complex. A total of $3 \mu \mathrm{l}$ of the GPR158-RGS7-G 35 complex was treated with $0.1 \%$ $\mathrm{OG}$ and applied to glow-discharged holey carbon grids (C-flat 1.2/1.3 Cu 400-mesh grid; EMS). The grids were plunge-frozen in liquid ethane using a Vitrobot Mark IV (Thermo Fisher Scientific) with a blot force of 3 and blot time of $5 \mathrm{~s}$ at $100 \%$ humidity and $4{ }^{\circ} \mathrm{C}$.

Five datasets were collected using a Talos Arctica electron microscope (FEI) operated at $200 \mathrm{kV}$ and equipped with a Gatan K3 summit direct electron detector in counting mode (Photon Science Center at Pohang University of Science and Technology) at a nominal magnification of $\times 79,000$, corresponding to $1.07 \AA /$ pixel. For the first dataset, 2400 movies were collected with a dose rate of 9.42 electrons per pixel per second for $6.07 \mathrm{~s}$, corresponding to a total dose of 50 electrons per $\AA^{2}$. For the second dataset, 2945 movies were collected with a dose rate of 10.422 electrons per pixel per second for $5.47 \mathrm{~s}$, corresponding to a total dose of 50 electrons per $\AA^{2}$. For the third dataset, 6497 movies were collected with a dose rate of 14.031 electrons per pixel per second for $4.08 \mathrm{~s}$, corresponding to a total dose of 50 electrons per $\AA^{2}$. For the fourth dataset, 5770 movies were collected with a dose rate of 10.077 electrons per pixel per second for $5.68 \mathrm{~s}$, corresponding to a total dose of 50 electrons per $\AA^{2}$. For the fifth dataset, 3,001 movies were collected with a dose rate of 17.762 electrons per pixel per second for $3.22 \mathrm{~s}$, corresponding to a total dose of 50 electrons per $\AA^{2}$. All datasets were collected using a defocus range of -1.0 to $-2.0 \mu \mathrm{m}$ over 50 frames.

Data processing for apo GPR158. Movie frames were aligned using MotionCor2 (ref. ${ }^{58}$ ), and motion-corrected sums were calculated to estimate the contrast transfer function (CTF) using CTFFIND4 (ref. ${ }^{59}$ ). Micrographs at low estimated resolution were removed, resulting in 8901 micrographs for data processing. A total of 5,152,593 particles were automatically picked in RELION-3 (ref. ${ }^{60}$ ) and exported to CryoSPARC v2.15 (ref. ${ }^{61}$ ). Particles were extracted with a box size of 310 pixels and subjected to several rounds of two-dimensional (2D) classification to eliminate contaminants and false-positive particles. Well-defined 2D classes were selected, resulting in 1,116,476 particles for further processing. Ab initio reconstruction and heterogeneous refinement in CryoSPARC v2.15 divided the particles into six classes. A dominant class (48.6\%) containing 543,174 particles showed a clear shape for both extracellular and TM domains, and was exported to RELION-3. The exported particles were improved via Bayesian polishing and CTF refinement, followed by another round of 3D classification without alignment. The final dataset of 425,819 particles (78.4\%) was subjected to 3D refinement and postprocessing without symmetry, which yielded a map with a global resolution of $3.52 \AA$ according to a FSC criterion of 0.143. A divided class with 180,744 particles in cryoSPARC v2.15 revealed a shorter conformation with a more compact middle linker. The particles were exported to RELION-3 and subjected to subsequent 3D refinement and postprocessing without symmetry, which resulted in a resolution of $5.47 \AA$. The final maps were sharpened using negative $B$-factors automatically determined by RELION-3.

\section{Data processing for the GPR158-RGS7-G $\beta 5$ complex. For the}

GPR158-RGS7-G 35 complexes, movies from five datasets were aligned and doseweighted using MotionCor2 (ref. ${ }^{58}$ ), and CTF parameters were calculated using CTFFIND4 (ref. ${ }^{59}$ ). After auto-picking the particles using template picker, 1,022,357 particles from 2349 micrographs (dataset 1), 1,147,777 particles from 2853 micrographs (dataset 2), 2,919,355 particles from 6221 micrographs (dataset 3), 1,347,653 particles from 4252 micrographs (dataset 4), and 873,753 particles from 2466 micrographs (dataset 5) were extracted individually using CryoSPARC v3.1 (ref. ${ }^{61}$ ). The combined particles from all datasets were subjected to several rounds of 2D classification using CryoSPARC v3.1. After exclusion of poorly defined classes, 1,971,816 particles were subjected to ab initio reconstruction to produce an initial 3D model using cryoSPARC v3.1. After heterogeneous refinement, 746,766 particles of one class showing the complex with RGS7-G $\beta 5$ were selected for further $\mathrm{ab}$ initio reconstruction using cryoSPARC v3.1. After performing heterogeneous refinement, 411,864 particles for the 2GPR158-RGS7-G $\beta 5$ complex were selected and subjected to two rounds of heterogeneous refinement. In total, 264,464 particles for the 2:1:1 complex were subjected to non-uniform refinement, local motion correction, and global CTF refinement. The processed particles were subjected to global non-uniform refinement yielding a map with a global resolution of $4.31 \AA$ A. The resulting map was subjected to particle subtraction to exclude the extracellular domain signal, followed by local non-uniform refinement using a mask on 7TM-RGS7-G 35 , generating a $4.3 \AA$ map. For the 2:2:2 complex, 375,895 particles with a 2:2:2 complex feature from each heterogeneous refinement were merged and subjected to local motion correction followed by heterogeneous refinement. This generated a map with a global resolution of $4.68 \AA$ To improve the map for two RGS7-G $\beta 5$ heterodimers and the cytoplasmic helices, particle subtraction was performed to exclude the density for the receptor and detergent micelle. The resulting particles including residual density were subjected to local non-uniform refinement using a mask for two RGS7-G $\beta 5$ heterodimers and the cytoplasmic helices, which produced a map with a global resolution of 4.61 Å. Additionally, to understand dynamics of RGS7-G $\beta 5$ in the 2:2:2 complex, the 3 D variability analysis ${ }^{62}$ in cryoSPARC v3.1 was performed using the 375,895 particles generated from non-uniform refinement: the calculation was focused on density including the cytoplasmic coiled-coil and two RGS7-G $\beta 5$ complexes with a filter resolution of $9 \AA$. A movie showing conformational variability was generated using UCSF Chimera v1.15 (ref. ${ }^{63}$ ) (Supplementary Movie 1).

Model building. The atomic model of apo GPR158 was built from the $3.52 \AA$ EM map. Main chain connectivity and secondary structural features were clearly resolved, and we placed $\alpha$-helices, $\beta$-strands, and linking loops of both extracellular and TM domains using $\mathrm{COOT}^{64}$. The map of the $\mathrm{CR}$, TM regions, and dimeric interface of the extracellular domain was of high quality, which allowed us to assign all side chains on the placed model. Assignment of side chains was guided by small residues (Gly, Ser, and Val) and bulky residues (Tyr, Phe, and Trp). No clear density was observed for side chains of the PAS domain except for helices $a 4$ and $a 5$; hence, these regions were built as poly-Ala chains. The model was subjected to real-space refinement using PHENIX 1.15.2 with geometry and secondary structural restraints ${ }^{65,66}$. The refined model has a MolProbity ${ }^{67}$ score of 1.74 and a clash-score of 3.54 (Supplementary Table 1).

The reported structure of Bos taurus RGS7-Mus musculus G $\beta 5$ (PDB 6N9G) ${ }^{47}$ was used as an initial template to build the 2GPR158-RGS7-GB5 model. Three residues differ between the human proteins and each of the RGS7 and G $\beta 5$ proteins from the initial template, and these residues were substituted to the equivalent human residues using COOT; Val291Leu, Ala298Leu and Arg375Lys in RGS7, and Asn34Ser, Asp46Glu and Val284Ile in GB5. The structures of human RGS7-GB5 and apo GPR158 were placed into the $4.3 \AA$ EM map using the fit-in-map tool of UCSF Chimera v1.14 (ref. ${ }^{63}$ ). Two turns of TM3 and ICL2 disordered in apo GPR158 were well-defined in the EM map of the complex, into which we manually built a model using COOT. The $\mathrm{Ha}$ and $\mathrm{Hb}$ helices were built as poly-Ala chains based on the EM maps. Residue numbers were assigned based on a recent publication ${ }^{7,8}, 3 \mathrm{D}$ structure prediction using the I-TASSER server ${ }^{44}$, and secondary structure prediction using PSIPRED v4.0 (ref. ${ }^{68}$ ) and XtalPred $\mathrm{RF}^{69}$. The 2:1:1 model was subjected to realspace refinement using PHENIX 1.15.2 with geometry and secondary structural restraints ${ }^{65,66}$. The refined model exhibited a MolProbity ${ }^{67}$ score of 2.13 and a clashscore of 9.65 (Supplementary Table 1). The following regions are not present in the map and not modeled: residues 1-17 (DEP), 219-255 (GGL), 450-495 (RGS) of 
RGS7, and residues 1-14 and 354-395 of G $\beta 5$. For the 2:2:2 complex, we docked the structures of GPR158 and human RGS7-G $\beta 5$ into the cryo-EM map using the fit-inmap tool of UCSF Chimera ${ }^{63}$ (Supplementary Fig. 9i, p).

Adenylate cyclase activation cell-based assay. The adenylate cyclase activation assay was performed following the procedure from the previous study ${ }^{11}$. GBR1GFP, GBR2-Flag, GPR158-Flag, RGS7-Flag, and G $\beta 5$ were cloned into the pEG BacMam vector (a gift from Dr. Eric Gouaux). All GPR158 mutations in this study were introduced by PCR-based site-directed mutagenesis and verified by DNA sequencing. Primer sequences are provided in Supplementary Table 2.

HEK293T cells were seeded at a density of $0.5 \times 10^{6}$ cells per well in a six-well plate containing complete growth medium, which consisted of high glucose DMEM (Lonza) and 10\% fetal bovine serum (FBS; Welgene), on the day before transfection. HEK293T cells were transfected with $5 \mu \mathrm{g}$ plasmids encoding GBR1-GFP, GBR2-Flag, RGS7-Flag, G $\beta 5$, GPR158-Flag, and pGloSensor-22F cAMP (Promega) using Fugene HD (Promega). Transfected cells were treated with $1 \mu \mathrm{M}$ GABA (Tocris Bioscience) for $24 \mathrm{~h}$. Cells were detached with Cell Dissociation Buffer (Gibco) and resuspended in equilibration medium, which comprised $86 \% \mathrm{CO}_{2}-$ independent medium (Invitrogen), 10\% FBS, 4\% GloSensor cAMP Reagent solution (Promega), and $1 \mu \mathrm{M}$ GABA. Cells in the equilibration medium were seeded in a tissue culture treated solid white 96 -well plate at a density of 100,000 cells per well and incubated for $2.5 \mathrm{~h}$ at room temperature. Expression levels of GBR1-GFP, GBR2-Flag, GPR158-Flag, and RGS7-Flag were examined by western blot analyses. Bioluminescence in the presence of $1 \mu \mathrm{M}$ GABA was initially quantified using a Centro XS ${ }^{3}$ LB 960 microplate luminometer (Berthold Technologies) with an integration time of $1 \mathrm{~s}$. After addition of $250 \mu \mathrm{M}$ CGP54626 (Tocris Bioscience) in $\mathrm{CO}_{2}$-independent medium, luminescence was subsequently measured every $90 \mathrm{~s}$. The results were normalized to fold changes between values obtained in the absence and presence of CGP54626. Statistical analysis was performed using GraphPad Prism 9.1.1. A one-way analysis of variance (ANOVA) and a multiple comparison correction of Tukey's post hoc test were used to analyze significant differences among conditions.

Fluorescent size exclusion chromatography analysis. Oligomeric state of the dimeric interface-disrupting mutant (F135A/F540A/W578A) was analyzed using fluorescent size exclusion chromatography. Genes encoding wild type or mutant full-length GPR158 were fused to GFP and inserted into pEG BacMam vector. The resulting plasmids were transfected into HEK293S $\mathrm{GnTI}^{-}$cells which were cultured using the protocol of Goehring et al. ${ }^{57}$. The cells were harvested and solubilized in the buffer containing $20 \mathrm{mM}$ HEPES pH 7.5, $300 \mathrm{mM} \mathrm{NaCl}, 1 \mathrm{mM}$ EDTA, $2 \%$ OG, and $0.2 \%$ CHS. After incubating for $1 \mathrm{~h}$ at $4{ }^{\circ} \mathrm{C}$, the solubilized membranes were cleared using ultracentrifugation at $99,278 \times g$ with a TLA45 rotor (Beckman) for $1 \mathrm{~h}$. The supernatants were loaded onto a Superose 6 10/300 column equilibrated with buffer comprising $20 \mathrm{mM}$ HEPES pH 7.5, $200 \mathrm{mM} \mathrm{NaCl}, 1 \mathrm{mM}$ EDTA, and $0.05 \%$ DDM. The eluent was detected by a fluorometer with excitation of $488 \mathrm{~nm}$ and emission of $512 \mathrm{~nm}$.

Subcellular fractionation. Cells transfected for the adenylate cyclase activation cell-based assay were subjected to the subcellular fractionation to compare expression levels of total, membrane, and cytosolic fractions of GBR1, GBR2, GPR158, and RGS7. The subcellular fractionation was carried out using the procedure from the previous study with minor modifications ${ }^{7}$. Cells were lysed in lysis buffer comprising $50 \mathrm{mM}$ Tris- $\mathrm{HCl} \mathrm{pH} \mathrm{7.4,150} \mathrm{mM} \mathrm{NaCl}, 1 \mathrm{mM}$ EDTA, $2.5 \mathrm{mM}$ $\mathrm{MgCl}_{2}$, and protease inhibitor cocktail (Roche) by sonication. Equal amounts of lysates were ultracentrifuged at $98,384 \times g$ with a TLA-55 rotor (Beckman) for $30 \mathrm{~min}$ at $4{ }^{\circ} \mathrm{C}$. The supernatant containing the cytosolic fraction was transferred to a fresh tube. The pellet was thoroughly washed with the lysis buffer and then incubated in $50 \mathrm{mM}$ Tris- $\mathrm{HCl} \mathrm{pH} \mathrm{7.4,300} \mathrm{mM} \mathrm{NaCl}, 1 \%$ Triton X-100, and protease inhibitor cocktail (Roche) for $40 \mathrm{~min}$ at $4{ }^{\circ} \mathrm{C}$. The membrane fraction was isolated by ultracentrifugation at $98,384 \times g$ for $15 \mathrm{~min}$. The lysates in the lysis buffer were supplemented with $1 \%$ Triton X-100 and incubated on ice for $1 \mathrm{~h}$. The total fraction was cleared by ultracentrifugation at $98,384 \times g$ for $30 \mathrm{~min}$. For western blot analyses, each fraction sample was resolved by $12 \%$ SDS-PAGE and transferred to an Immobilon-P PVDF membrane (Sigma). The following antibodies were diluted to the manufacturers' working concentration and incubated with the membranes for $14 \mathrm{~h}$ at $4{ }^{\circ} \mathrm{C}$; anti-OctA antibody (Santa Cruz Biotechnology, \#sc-166355, 1:400 dilution), anti-GFP antibody (Santa Cruz Biotechnology, \#sc-9996, 1:40 dilution), anti-glyceraldehyde-3-phosphate dehydrogenase antibody (GAPDH; Sigma, \#MAB374, 1:5000 dilution) and anti-Ncadherin antibody (Sigma, \#C3865, 1:50). Anti-OctA antibody was used to detect Flag tags from GBR2, GPR158 and RGS7, and anti-GFP antibody was for GBR1. After incubation with anti-mouse IgG secondary antibodies (Santa Cruz Biotechnology, \#c-516102, 1:2000 dilution) conjugated with horseradish peroxidase at room temperature for $2 \mathrm{~h}$, signals were developed using SuperSignal West Femto Maximum Sensitivity Substrate (Thermo Fisher Scientific) and detected with a Amersham Imager 680 chemiluminescence imaging system (GE Healthcare). Statistical analysis was performed using GraphPad Prism 9.1.1. A one-way analysis of variance (ANOVA) test and a multiple comparison correction of Tukey's post hoc test were used to analyze significant differences among conditions. Uncropped and unprocessed scan blots are included in the Source data file

Reporting summary. Further information on research design is available in the Nature Research Reporting Summary linked to this article.

\section{Data availability}

Atomic coordinates and the cryo-EM map have been deposited in the PDB and the EM Data Bank, respectively, under following accession numbers: EMD-31351 and 7EWL (apo GPR158), EMD-31360 (overall refined 2GPR158-RGS7-GB5), EMD-31365 (locally refined 2GPR158-RGS7-GB5), and 7EWP (2GPR158-RGS7-GB5), and EMD-31363 (overall refined 2GPR158-2RGS7-2Gß35), EMD-31366 (locally refined 2GPR158-2RGS72GB5), and 7EWR (2GPR158-2RGS7-2G 35 ). The reported structural model of Bos taurus RGS7-Mus musculus G $\beta 5$ (PDB $6 \mathrm{~N} 9 \mathrm{G}$ ) ${ }^{47}$ was used as an initial template to build the 2GPR158-RGS7-G $\beta 5$ model. Source data are provided with this paper.

Received: 22 June 2021; Accepted: 4 November 2021; Published online: 23 November 2021

\section{References}

1. Orlandi, C. et al. GPR158/179 regulate G protein signaling by controlling localization and activity of the RGS7 complexes. J. Cell Biol. 197, 711-719 (2012).

2. Khrimian, L. et al. Gpr158 mediates osteocalcin's regulation of cognition. J. Exp. Med. 214, 2859-2873 (2017).

3. Sutton, L. P. et al. Orphan receptor GPR158 controls stress-induced depression. Elife 7, e33273 (2018).

4. Condomitti, G. et al. An input-specific orphan receptor GPR158-HSPG interaction organizes hippocampal Mossy fiber-CA3 synapses. Neuron 100, 201-215 e209 (2018).

5. Patel, N. et al. Expression and functional role of orphan receptor GPR158 in prostate cancer growth and progression. PLoS ONE 10, e0117758 (2015).

6. Chun, L., Zhang, W. H. \& Liu, J. F. Structure and ligand recognition of class C GPCRs. Acta Pharmacol. Sin. 33, 312-323 (2012).

7. Orlandi, C. et al. Orphan receptor GPR158 is an allosteric modulator of RGS7 catalytic activity with an essential role in dictating its expression and localization in the brain. J. Biol. Chem. 290, 13622-13639 (2015).

8. Hajj, M. et al. Nonclassical ligand-independent regulation of Go protein by an orphan Class C G-protein-coupled receptor. Mol. Pharmacol. 96, 233-246 (2019).

9. Watkins, L. R. \& Orlandi, C. In vitro profiling of orphan G protein coupled receptor (GPCR) constitutive activity. Br. J. Pharmacol. 178, 2963-2975 (2021).

10. Ostrovskaya, O. I. et al. Inhibitory signaling to ion channels in hippocampal neurons is differentially regulated by alternative macromolecular complexes of RGS7. J. Neurosci. 38, 10002-10015 (2018).

11. Orlandi, C., Sutton, L. P., Muntean, B. S., Song, C. \& Martemyanov, K. A. Homeostatic cAMP regulation by the RGS7 complex controls depressionrelated behaviors. Neuropsychopharmacology 44, 642-653 (2019).

12. McCoy, K. L. \& Hepler, J. R. Regulators of G protein signaling proteins as central components of $\mathrm{G}$ protein-coupled receptor signaling complexes. Prog. Mol. Biol. Transl. Sci. 86, 49-74 (2009).

13. Sandiford, S. L. \& Slepak, V. Z. The Gbeta5-RGS7 complex selectively inhibits muscarinic $\mathrm{M} 3$ receptor signaling via the interaction between the third intracellular loop of the receptor and the DEP domain of RGS7. Biochemistry 48, 2282-2289 (2009).

14. Hague, C. et al. Selective inhibition of alpha1A-adrenergic receptor signaling by RGS2 association with the receptor third intracellular loop. J. Biol. Chem. 280, 27289-27295 (2005)

15. Miyamoto-Matsubara, M., Saitoh, O., Maruyama, K., Aizaki, Y. \& Saito, Y. Regulation of melanin-concentrating hormone receptor 1 signaling by RGS8 with the receptor third intracellular loop. Cell Signal. 20, 2084-2094 (2008).

16. Masuho, I., Xie, K. \& Martemyanov, K. A. Macromolecular composition dictates receptor and $\mathrm{G}$ protein selectivity of regulator of $\mathrm{G}$ protein signaling (RGS) 7 and 9-2 protein complexes in living cells. J. Biol. Chem. 288, 25129-25142 (2013)

17. Georgoussi, Z. et al. Selective interactions between $G$ protein subunits and RGS4 with the C-terminal domains of the mu- and delta-opioid receptors regulate opioid receptor signaling. Cell Signal. 18, 771-782 (2006).

18. Chen, S. H. \& Hamm, H. E. DEP domains: more than just membrane anchors. Dev. Cell 11, 436-438 (2006).

19. O’Brien, J. B., Wilkinson, J. C. \& Roman, D. L. Regulator of G-protein signaling (RGS) proteins as drug targets: Progress and future potentials. J. Biol. Chem. 294, 18571-18585 (2019). 
20. Holm, L. \& Rosenstrom, P. Dali server: conservation mapping in 3D. Nucleic Acids Res. 38, W545-W549 (2010).

21. Moglich, A., Ayers, R. A. \& Moffat, K. Structure and signaling mechanism of Per-ARNT-Sim domains. Structure 17, 1282-1294 (2009).

22. Liu, Y. C., Machuca, M. A., Beckham, S. A., Gunzburg, M. J. \& Roujeinikova, A. Structural basis for amino-acid recognition and transmembrane signalling by tandem Per-Arnt-Sim (tandem PAS) chemoreceptor sensory domains. Acta Crystallogr. D Biol. Crystallogr. 71 (2015).

23. Zhang, Z. \& Hendrickson, W. A. Structural characterization of the predominant family of histidine kinase sensor domains. J. Mol. Biol. 400, 335-353 (2010).

24. Rao, Z. et al. The structure of a $\mathrm{Ca}(2+)$-binding epidermal growth factor-like domain: its role in protein-protein interactions. Cell 82, 131-141 (1995).

25. Tan, K., Duquette, M., Joachimiak, A. \& Lawler, J. The crystal structure of the signature domain of cartilage oligomeric matrix protein: implications for collagen, glycosaminoglycan and integrin binding. FASEB J. 23, 2490-2501 (2009).

26. Carlson, C. B. et al. Structure of the calcium-rich signature domain of human thrombospondin-2. Nat. Struct. Mol. Biol. 12, 910-914 (2005).

27. Pak, J. S. et al. NELL2-Robo3 complex structure reveals mechanisms of receptor activation for axon guidance. Nat. Commun. 11, 1489 (2020).

28. Ballesteros, J. A. \& Weinstein, H. Integrated methods for the construction of three-dimensional models and computational probing of structure-function relations in G protein-coupled receptors. Methods Neurosci. 25, 366-428 (1995).

29. Pin, J. P., Galvez, T. \& Prezeau, L. Evolution, structure, and activation mechanism of family 3/C G-protein-coupled receptors. Pharmacol. Ther. 98, 325-354 (2003).

30. Koehl, A. et al. Structural insights into the activation of metabotropic glutamate receptors. Nature 566, 79-84 (2019).

31. Park, J. et al. Structure of human GABAB receptor in an inactive state. Nature 584, 304-309 (2020).

32. Papasergi-Scott, M. M. et al. Structures of metabotropic GABAB receptor. Nature 584, 310-314 (2020).

33. Kim, Y., Jeong, E., Jeong, J. H., Kim, Y. \& Cho, Y. Structural basis for activation of the heterodimeric GABAB receptor. J. Mol. Biol. 432, 5966-5984 (2020).

34. Mao, C. et al. Cryo-EM structures of inactive and active $\mathrm{GABA}_{\mathrm{B}}$ receptor. Cell Res. 30, 564-573 (2020).

35. Shaye, $H$. et al. Structural basis of the activation of a metabotropic GABA receptor. Nature 584, 298-303 (2020).

36. Shen, C. et al. Structural basis of GABAB receptor-Gi protein coupling. Nature 594, 594-598 (2021)

37. Ling, S. et al. Structural mechanism of cooperative activation of the human calcium-sensing receptor by $\mathrm{Ca}(2+)$ ions and L-tryptophan. Cell Res. 31, 383-394 (2021).

38. Stauch, B. et al. Structural basis of ligand recognition at the human MT1 melatonin receptor. Nature 569, 284-288 (2019).

39. Lin, X. et al. Structural basis of ligand recognition and self-activation of orphan GPR52. Nature 579, 152-157 (2020).

40. Nass, $\mathrm{K}$. et al. Advances in long-wavelength native phasing at X-ray freeelectron lasers. IUCrJ 7, 965-975 (2020).

41. Liu, X. et al. An allosteric modulator binds to a conformational hub in the beta2 adrenergic receptor. Nat. Chem. Biol. 16, 749-755 (2020).

42. Dore, A. S. et al. Structure of class C GPCR metabotropic glutamate receptor 5 transmembrane domain. Nature 511, 557-562 (2014).

43. $\mathrm{Wu}, \mathrm{H}$. et al. Structure of a class C GPCR metabotropic glutamate receptor 1 bound to an allosteric modulator. Science 344, 58-64 (2014).

44. Zhang, Y. I-TASSER server for protein 3D structure prediction. BMC Bioinformatics 9, 40 (2008).

45. Seven, A. B. et al. G-protein activation by a metabotropic glutamate receptor. Nature 595, 450-454 (2021).

46. Lin, S. et al. Structures of Gi-bound metabotropic glutamate receptors mGlu2 and mGlu4. Nature 594, 583-588 (2021).

47. Patil, D. N. et al. Structural organization of a major neuronal G protein regulator, the RGS7-Gbeta5-R7BP complex. Elife 7, e42150 (2018).

48. Henry, J. T. \& Crosson, S. Ligand-binding PAS domains in a genomic, cellular, and structural context. Annu. Rev. Microbiol. 65, 261-286 (2011).

49. McIntosh, B. E., Hogenesch, J. B. \& Bradfield, C. A. Mammalian Per-Arnt-Sim proteins in environmental adaptation. Annu. Rev. Physiol. 72, 625-645 (2010).

50. Maurice, $P$. et al. Molecular organization and dynamics of the melatonin MT(1) receptor/RGS20/G(i) protein complex reveal asymmetry of receptor dimers for RGS and G(i) coupling. EMBO J. 29, 3646-3659 (2010).

51. Ross, E. M. \& Wilkie, T. M. GTPase-activating proteins for heterotrimeric G proteins: regulators of G protein signaling (RGS) and RGS-like proteins. Annu. Rev. Biochem 69, 795-827 (2000).

52. Masuho, I. et al. Global map of $\mathrm{G}$ protein signaling regulation by RGS proteins. Cell 83, 503-521 (2020).
53. Xie, G. X. \& Palmer, P. P. How regulators of G protein signaling achieve selective regulation. J. Mol. Biol. 366, 349-365 (2007).

54. Ballon, D. R. et al. DEP-domain-mediated regulation of GPCR signaling responses. Cell 126, 1079-1093 (2006).

55. Cheever, M. L. et al. Crystal structure of the multifunctional Gbeta5-RGS9 complex. Nat. Struct. Mol. Biol. 15, 155-162 (2008).

56. Velazhahan, V. et al. Structure of the class D GPCR Ste2 dimer coupled to two G proteins. Nature 589, 148-153 (2021).

57. Goehring, A. et al. Screening and large-scale expression of membrane proteins in mammalian cells for structural studies. Nat. Protoc. 9, 2574-2585 (2014).

58. Zheng, S. Q. et al. MotionCor2: anisotropic correction of beam-induced motion for improved cryo-electron microscopy. Nat. Methods 14, 331-332 (2017).

59. Rohou, A. \& Grigorieff, N. CTFFIND4: fast and accurate defocus estimation from electron micrographs. J. Struct. Biol. 192, 216-221 (2015).

60. Zivanov, J. et al. New tools for automated high-resolution cryo-EM structure determination in RELION-3. Elife 7, 42166 (2018).

61. Punjani, A., Rubinstein, J. L., Fleet, D. J. \& Brubaker, M. A. cryoSPARC algorithms for rapid unsupervised cryo-EM structure determination. Nat. Methods 14, 290-296 (2017).

62. Punjani, A. \& Fleet, D. J. 3D variability analysis: resolving continuous flexibility and discrete heterogeneity from single particle cryo-EM. J. Struct. Biol. 213, 107702 (2021).

63. Pettersen, E. F. et al. UCSF Chimera-a visualization system for exploratory research and analysis. J. Comput Chem. 25, 1605-1612 (2004).

64. Emsley, P. \& Cowtan, K. Coot: model-building tools for molecular graphics Acta Crystallogr. D Biol. Crystallogr. 60, 2126-2132 (2004).

65. Adams, P. D. et al. Recent developments in the PHENIX software for automated crystallographic structure determination. J. Synchrotron Radiat. 11, 53-55 (2004).

66. Afonine, P. V. et al. Real-space refinement in PHENIX for cryo-EM and crystallography. Acta Crystallogr. D Struct. Biol. 74, 531-544 (2018).

67. Chen, V. B. et al. MolProbity: all-atom structure validation for macromolecular crystallography. Acta Crystallogr. D. Biol. Crystallogr. 66, $12-21(2010)$

68. Buchan, D. W., Minneci, F., Nugent, T. C., Bryson, K. \& Jones, D. T. Scalable web services for the PSIPRED Protein Analysis Workbench. Nucleic Acids Res. 41, W349-W357 (2013)

69. Slabinski, L. et al. XtalPred: a web server for prediction of protein crystallizability. Bioinformatics 23, 3403-3405 (2007).

\section{Acknowledgements}

We thank J.S. Ko for assistance in cloning, and Y.J. Kim and members of the Cho's lab for comments. This work was supported by grants from the National Research Foundation of Korea (NRF) funded by the Korea government (MEST, No.

2021R1A2C301335711 and 2017M3A9F6029736), Samsung Science and Technology Foundation (SSTF-BA1602-14), BK21 program (Ministry of Education) to Y.C. E.J. and Y.K. acknowledge financial support from POSTECH Basic Science Research Institute Grant (2021R1A6A1A10042944).

\section{Author contributions}

E.J. and Y.K. carried out protein expression, purification, and structure determination; E.J., Y.K. and J.J. participated in biochemical experiments; E.J, Y.K., J.J. and Y.C. designed research; and E.J, Y.K. and Y.C. wrote the manuscript

\section{Competing interests}

The authors declare no competing interests.

\section{Additional information}

Supplementary information The online version contains supplementary material available at https://doi.org/10.1038/s41467-021-27147-1.

Correspondence and requests for materials should be addressed to Yunje Cho.

Peer review information Nature Communications thanks Matthew Belousoff and the other, anonymous, reviewer(s) for their contribution to the peer review of this work. Peer reviewer reports are available.

Reprints and permission information is available at http://www.nature.com/reprints

Publisher's note Springer Nature remains neutral with regard to jurisdictional claims in published maps and institutional affiliations. 
(c) (i) Open Access This article is licensed under a Creative Commons Attribution 4.0 International License, which permits use, sharing, adaptation, distribution and reproduction in any medium or format, as long as you give appropriate credit to the original author(s) and the source, provide a link to the Creative Commons license, and indicate if changes were made. The images or other third party material in this article are included in the article's Creative Commons license, unless indicated otherwise in a credit line to the material. If material is not included in the article's Creative Commons license and your intended use is not permitted by statutory regulation or exceeds the permitted use, you will need to obtain permission directly from the copyright holder. To view a copy of this license, visit http://creativecommons.org/ licenses/by/4.0/.

(C) The Author(s) 2021 